\title{
A STUDY ON PLANNING AND PLATFORM FOR INTERACTIVE EXHIBITION OF SCIENTIFIC CULTURAL HERITAGE
}

\author{
Y. H. Jo ${ }^{1,}{ }^{*}$, J. Kim $^{2}$, N. C. Cho ${ }^{1}$, C. H. Lee ${ }^{1}$, Y. H. Yun ${ }^{3}$, D. K. Kwon ${ }^{1}$ \\ ${ }^{1}$ Dept. of Cultural Heritage Conservation Sciences, Kongju National University, 32588, Republic of Korea \\ - (joyh, nam1611, chanlee)@kongju.ac.kr,(dkdk0831)@naver.com \\ Technology Research Institute for Cultural Heritage - (jikio)@trich.or.kr \\ Exhibition Division, National Science Museum - (auspice)@korea.kr
}

Commission II, WG II/8

KEY WORDS: Bronze Mirrors, Interactive Projection mapping, Scientific Culture Heritage, Media Art, Exhibition Platform

\begin{abstract}
:
This study has developed an experiential exhibition system of scientific cultural heritage through interactive projection mapping. A bronze mirror with fine linear design was selected as an exhibition subject considering the intuitiveness and responsiveness of research and development results. First, three-dimensional (3D) scanning was performed to construct a digital database of the original forms, and computer graphics-based 3D modeling was also performed to build exhibition materials and 3D printed objects. Furthermore, objective scenarios were created based on a traditional science experience, understanding the present bronze mirror, and processes of restoring the corroded original bronze mirror through modern conservation science. The technique selected to implement the scientific content of bronze mirror as media art in this study is interactive projection mapping. Moreover, a large 3D printing of bronze mirror was installed at the center of the exhibition space to allow users and all viewers to concurrently engage in scientific cultural heritage content. Simultaneous projection mapping on the front and rear of the printed bronze mirror model enhanced 3D effects and the immersion of the content. To construct an interactive space connecting the virtual and reality, ore, mold, bronze mirror replicas, 3D printing outputs, and cultural products used in the process of producing bronze mirrors were exhibited to make the exhibit more intuitive for visitors. This interactive projection mapping-based scientific and cultural exhibition of the bronze mirror is an experiential exhibition system which combines traditional science, modern conservation science, and future exhibition technology.
\end{abstract}

\section{INTRODUCTION}

Many countries around the world have excellent scientific cultural heritage and heritage content development and exhibitions are also relatively active, focusing on science museums and art museums. However, existing exhibitions of scientific cultural heritage mainly use one-way communication using sound and video, which has a limited ability to attract viewer's interest or to immerse them. The one-way delivery of the exhibition content is insufficient to promote understanding of the science and technology of cultural heritage and may degrade visitor attraction and economic efficiency compared to investment cost in the long term.

Exhibitions of media art that combine digital technology and culture content have recently drawn attention along with the rapid development of the IT industry. Unlike existing exhibits that are unilaterally appreciated, this media art can enhance interaction with visitors and diversity of content through the transformation of culture and art objects. As a result, museums, art galleries, and science museums are actively utilizing media arts as a new exhibition technique (Krautsack, 2011; Yun et al., 2013; Nofal et al., 2018; Yamazoe et al., 2018).

Through interactive projection mapping, this study has developed an experiential exhibition system that enables visitors to intuitively understand the production process, corrosion mechanisms, scientific analyses, and conservation treatment processes of scientific cultural heritage (Jo et al., 2018). Among various materials and forms of scientific cultural heritage, a bronze mirror with fine linear design was selected as an exhibition subject considering the intuitiveness and responsiveness of research and development results. In other words, the convergence of the traditional science related to the production of bronze mirrors and the modern science that restores its function as a mirror is the core of this scientific and cultural exhibition.

\section{ESTABLISHMENT OF SCIENTIFIC CULTURAL HERITAGE CONTENT}

The bronze mirror was selected as a subject for scientific and cultural exhibition because it contains many convergent elements that can be utilized in media art exhibitions, including scientific, technological, engineering, mathematical, and artistic elements. In particular, a bronze mirror with fine linear design is one of the representative artifacts of Korea, and its reflectivity and sharpness are comparable to that of a modern mirror. Approximately 13,000 straight lines and concentric circles are drawn on a mirror about $20 \mathrm{~cm}$ in diameter, and the production techniques are a heritage mystery. However, as most bronze mirrors exhibited in the museum are corroded, visitors are often unable to recognize their functions. Accordingly, the technological process of producing and restoring the mirror's original form through modern conservation science are very suitable as scientific scenarios.

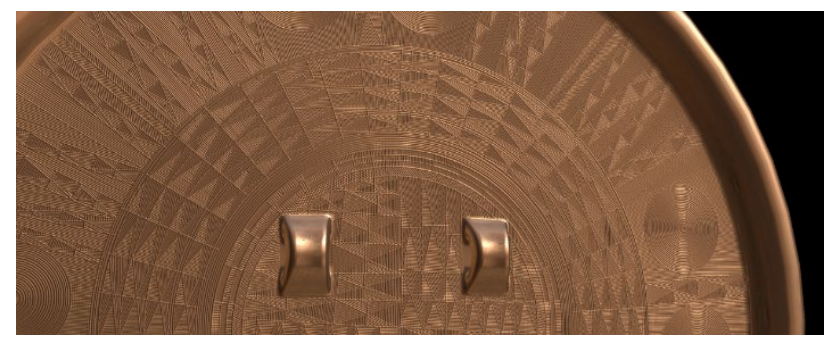

Figure 1. Computer graphics-based 3D modeling of the bronze mirror with fine linear design 
In this study, historical, art historic, and scientific data on bronze mirrors were collected and objective scenarios were created based on this data in order to increase the reliability of the content of scientific cultural heritage. Furthermore, three-dimensional (3D) scanning was performed to construct a digital database of the original forms. The study used a high-resolution optical scanner to create $3 \mathrm{D}$ models to which various rendering techniques were applied due to the fine linear design on the surfaces of the bronze mirror, which is currently managed in the Korean Christian Museum at Soongsil University. Particularly, computer graphics-based 3D modeling was also performed to build exhibition materials and 3D printed objects (Figure 1).
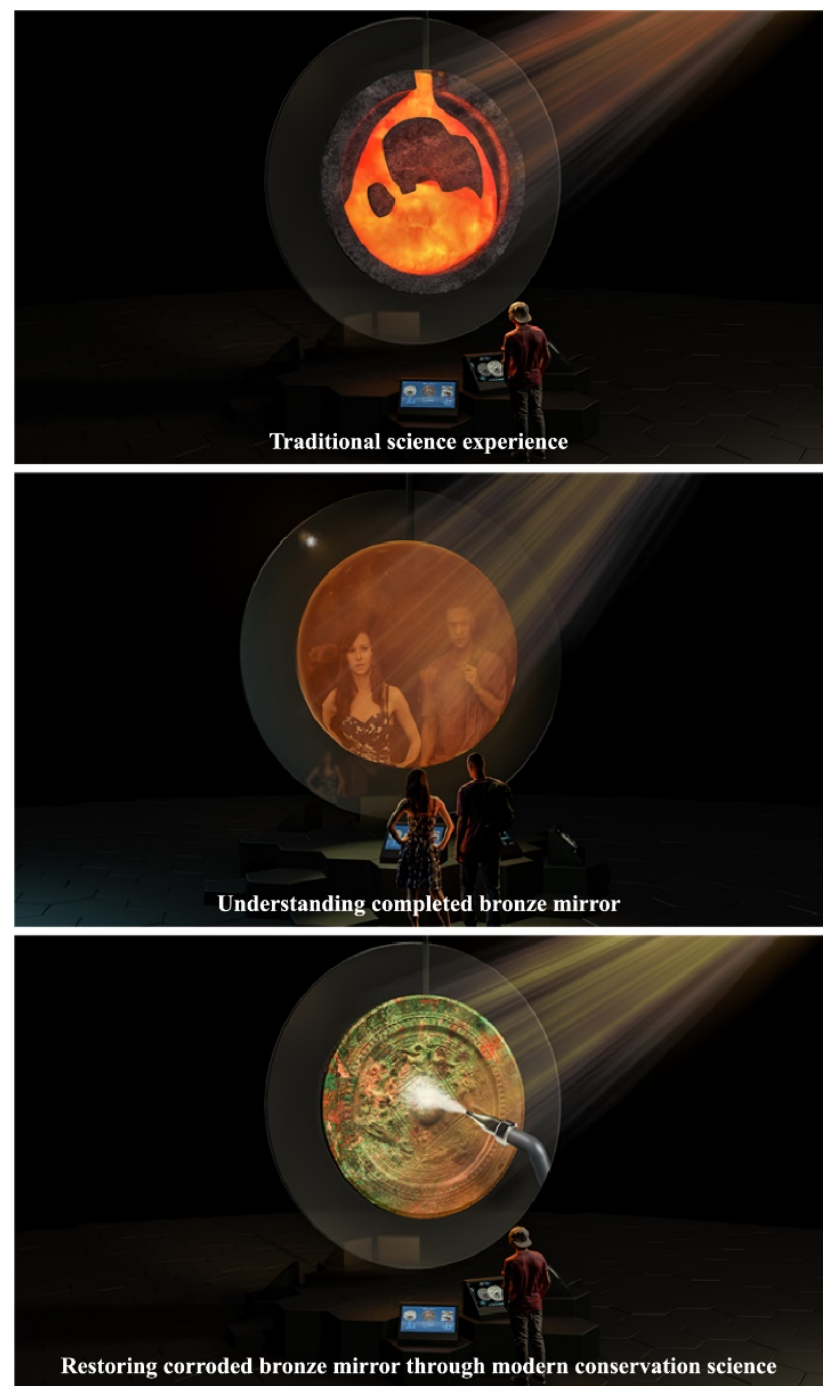

Figure 2. Media art scenarios of the bronze mirror

\section{CREATION OF MEDIA ART SCENARIO}

The first step of media art scenario for the bronze mirror is a traditional science experience in which a visitor makes a bronze mirror through mining, smelting, refining, casting, and cooling (Figure 2). In the scenario, visitors view each exhibit in the form of digital images through an interactive process and understand the mirror's function through the camera mirror system. The second step is to understand the present bronze mirror (Figure 2). When the user introduces a corrosive environment, a bronze mirror which no longer functions as a mirror appears. Displaying the mirror with no function enables the exhibition to maximize its educational effect.
The third step is the process of restoring the corroded original bronze mirror through modern cultural heritage conservation science technology (Figure 3). For this purpose, the visitor performs a scientific investigation with a microscope for interaction purposes and then experiences scientific conservation treatments, such as cleaning, joining, and consolidating. Once these missions are completed, finally, the historical content related to the prehistoric age is shown through the surface of the bronze mirror which restored the original form, and this gives a comprehensive understanding of the culture of the time.

\section{EXHIBITION SYSTEM AND SPACE DESIGN}

One of the important elements in science exhibition for the bronze mirror is to build a multi-sensory exhibition system and space design that can induce visitor immersion. The technique selected to implement the scientific content of bronze mirror as media art in this study is interactive projection mapping (Krautsack, 2011; Grundhöfer and Iwai, 2018; Guo et al., 2018). This technique makes it possible for users to intuitively understand the traditional science of the bronze mirror and the modern conservation science by interacting with the viewer's motion recognition in accordance with the content utilization method.

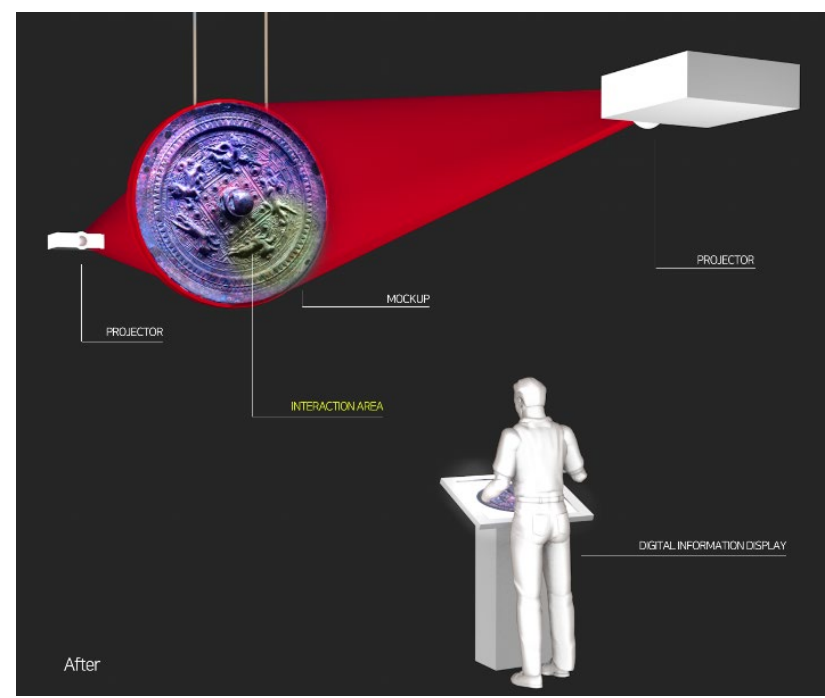

Figure 3. Schematic image of projection mapping for scientific cultural heritage exhibition of the bronze mirror

Moreover, a large 3D printing of bronze mirror was installed at the center of the exhibition space to allow users and all viewers to concurrently engage in scientific cultural heritage content. The $3 \mathrm{D}$ printing method is material extrusion using PLA and the size is about $1 \mathrm{~m}$. In particular, through the interaction of motion recognition, DID, and the projector, the scientific cultural heritage content is projected onto the printed bronze mirror model. That is, when a user's instructions are input with the guidance of the DID, special effects are displayed together with various content, and this allows users to experience various stepby-step missions related to traditional science and modern conservation science. Simultaneous projection mapping on the front and rear of the printed bronze mirror model enhanced 3D effects and the immersivity of the content (Figure 3).

The most important consideration in the design of the exhibition space is to construct an interactive space connecting the virtual 
and reality and to acquire immersion and objective information related to the content (Figure 4). For this purpose, ore, mold, bronze mirror replicas, 3D printing models, and cultural products used in the process of producing bronze mirrors were exhibited to make the exhibit more intuitive for visitors. In addition, making film and various rendering images related to bronze mirrors for research and development process were constructed to enhance the understanding of scientific cultural heritage content.

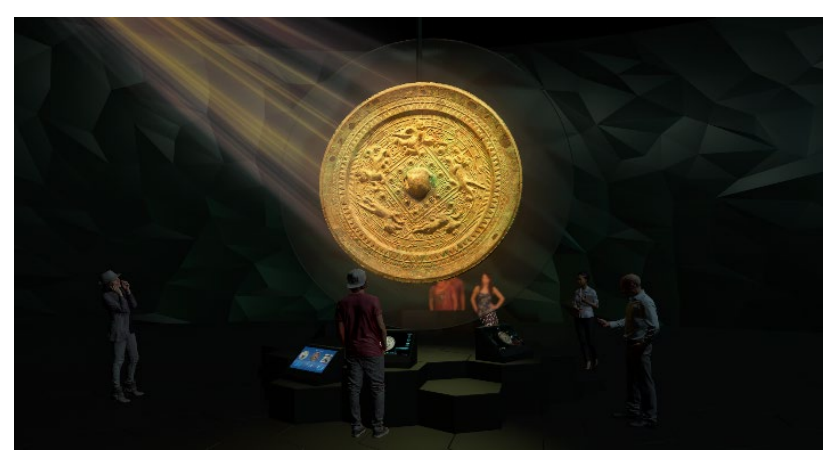

Figure 4. Space design of scientific exhibition system for the bronze mirror

\section{DISCUSSION AND CONCLUSION}

This study established scientific and cultural exhibition content that enables one to easily and intuitively understand scientific elements, such as production, corrosion, analysis, and conservation treatment processes of the bronze mirror. The combination of a potentially abstruse science exhibition focusing on principles and theories and media art with strong visual effects led the audience to active participation. It also developed an immersive presentation system to bring the exhibition platform to new heights.

This interactive projection mapping-based scientific and cultural exhibition of the bronze mirror is an experiential exhibition system which combines traditional science, modern conservation science, and future exhibition technology; hence it is highly valuable as a source technology. Particularly, it can be used as an important platform for expanding from museum exhibitions centered on ancient history and art history to scientific cultural heritage. In addition, bronze mirrors have excellent global universality, and thus, the exhibition can be extended to East Asian and European markets. It will also provide ample opportunities for Korea to encourage the popularization of its scientific and cultural heritage and take a leap into becoming a content powerhouse.

\section{ACKNOWLEDGEMENTS}

This research was supported by Scientific and Cultural Exhibition Service Program through the National Research Foundation of Korean (NRF) funded by the Ministry of Science, ICT (NRF-2018X1A3A1070017).

\section{REFERENCES}

Grundhöfer, A., Iwai, D., 2018. Recent Advances in Projection Mapping Algorithms, Hardware and Applications. Computer Graphics Forum, 37, 653-675.
Guo, Y., Chu, S.C., Liu, Z., Qiu, C., Luo, H., Tan, J., 2018. A real-time interactive system of surface reconstruction and dynamic projection mapping with RGB-depth sensor and projector. International Journal of Distributed Sensor Networks, $14,1-12$.

Jo, Y.H., Kim, J., Cho, N. C., Lee, C.H., Kwon, D.K., Yun, Y.H., 2018. A proposal of science exhibition system for bronze mirror through visitors reactive projection mapping. In: Proceedings of the International Symposium of Science Museums, 322-324.

Krautsack, 2011. 3D Projection Mapping and its Impact on Media \& Architecture in Contemporary and Future Urban Spaces. Journal of the New Media Caucus, 7.

Nofal, E., Stevens, R., Coomans, T., Moere, A.V., 2018. Communicating the spatiotemporal transformation of architectural heritage via an in-situ projection mapping installation. Digital Applications in Archaeology and Cultural Heritage, 10, e00083.

Yamazoe, H., Kasetani, M., Noguchi, T., Lee, J.H., 2018. Projection mapping onto multiple objects using a projector robot. Advances in Robotics Research, 2, 45-57.

Yun, H.R., Kim, D.W., Ishii, T., 2013. A Study of digital media art utilizing the contents of the architecture cultural property. International Journal of Asia Digital Art \& Design, 17, 77-84. 